This document is confidential and is proprietary to the American Chemical Society and its authors. Do not copy or disclose without written permission. If you have received this item in error, notify the sender and delete all copies.

\title{
Unfolding Dynamics of a Photo-Switchable Helical Peptide
}

\begin{tabular}{|c|c|}
\hline Journal: & The Journal of Physical Chemistry \\
\hline Manuscript ID & jp-2020-04017p.R1 \\
\hline Manuscript Type: & Article \\
\hline $\begin{array}{r}\text { Date Submitted by the } \\
\text { Author: }\end{array}$ & $\mathrm{n} / \mathrm{a}$ \\
\hline Complete List of Authors: & $\begin{array}{l}\text { Auvray, Francois; University of Nottingham, School of Chemistry } \\
\text { Hirst, Jonathan; University of Nottingham, School of Chemistry }\end{array}$ \\
\hline
\end{tabular}

\section{SCHOLARONE \\ Manuscripts}




\title{
Unfolding dynamics of a photo-switchable
} helical peptide

\author{
Francois Auvray and Jonathan D. Hirst* \\ School of Chemistry, University of Nottingham, University Park, Nottingham NG7 2RD, \\ U.K. \\ E-mail: jonathan.hirst@nottingham.ac.uk \\ Phone: +44 115951 3478. Fax: +44 1159513562
}

\begin{abstract}
We present an atomistic force field for the azo-moiety of the photo-switchable FK11-X peptide. We use the parameters to study the unfolding of the peptide through molecular dynamics simulations. The unfolded ensemble contains many different structures, ranging from a partially unfolded peptide to a fully unfolded structure. The averaged computed far-ultraviolet circular dichroism (CD) spectrum of the set of structures, which were simulated using the newly developed force field, agrees well with experiment. The rate of the simulated unfolding process was estimated to have a time constant of $5.80 \pm 0.03 \mathrm{~ns}$ from the time-evolution of the CD spectrum of the peptide, computed from the backbone conformations sampled over 40 simulated trajectories. Our estimated time constant is faster than, but not inconsistent with, previous experimental estimates from time-resolved infrared and optical rotatory dispersion spectroscopy.
\end{abstract}




\section{Introduction}

The cooperative nature of protein folding suggests that individual $\alpha$-helices should not fold in isolation. However, Brown and Klee ${ }^{1}$ observed, using circular dichroism (CD) spectroscopy, partial helical structure in the isolated C-peptide of ribonuclease A. Subsequently, the thermodynamics and kinetics of the folding of $\alpha$-helices have been widely studied ${ }^{2}$ and these studies have elucidated many important aspects of protein folding. One example of many was examination of the stabilizing thermodynamic influence of side-chain interactions in isolated helices in the absence of the complexities of a surrounding protein environment. ${ }^{3}$

The unfolding dynamics of helical peptides have been studied using temperature-jump (T-jump) techniques in conjunction with various spectroscopies. T-jump experiments move a system away from equilibrium by rapid heating of the solvent by several degrees within a few nanoseconds by irradiating the sample with a pulsed infrared laser. They provide insights on microsecond and nanosecond timescales, which are of particular interest for early events in protein folding. Techniques employed in these studies include infrared spectroscopy ${ }^{4}$ and ultraviolet (UV) resonance Raman spectroscopy. ${ }^{5}$ Khuc et al. ${ }^{6}$ used a T-jump farUV CD experiment with a time step of 12 ns to follow the loss of helical structure upon thermal denaturation of a poly(glutamic acid) peptide, exploiting a well-known empirical relationship between the intensity of a distinctive negative band at $222 \mathrm{~nm}$ and the $\alpha$ helical content. ${ }^{7}$ The influence of the backbone conformation on far-UV CD spectra has been explored theoretically over many years, ${ }^{8,9}$ including the role of main-chain hydrogen bond lengths ${ }^{10}$ and the sensitivity to the backbone dihedral angles. ${ }^{11}$ This provides a direct connection between atomistic models of peptide (un)folding and CD spectroscopy.

Molecular dynamics (MD) simulations on nanosecond and microsecond timescales complement experimental investigations and furnish atomistic detail about the folding mechanism and the associated folding landscape, including the transiently stable conformations. ${ }^{12,13}$ Simulations of peptides are computationally more tractable than simulations of proteins, because of their smaller size. However, this is somewhat offset by their greater conformational 

of the empirical force fields used in the simulations. Confidence in the sampling and the force field can be developed by computing spectroscopic observables, with the caveat that reproduction of experiment is a necessary but not sufficient condition for establishing the adequacy of the simulation methodology.

There are several approaches to computing CD spectra from the atomic coordinates from an MD simulation (or indeed an experimentally determined structure) of a peptide or protein. These have recently been reviewed in detail elsewhere. ${ }^{14}$ Empirical approaches, such as BeStSel, ${ }^{15}$ PDB2CD ${ }^{16}$ and SESCA, ${ }^{17}$ take various structural descriptors derived from the atomic coordinates and some "basis" spectra to generate a computed spectrum. Quantum chemical methods can be used. Time-dependent density functional theory (TDDFT) has been employed ${ }^{18}$ and inventive approaches applicable to large molecular systems have been proposed, ${ }^{19}$ involving simplified Tamm-Dancoff density functional theory.

Calculations based on exciton theory, ${ }^{20,21}$ with an effective Hamiltonian, are more approximate than fully quantum mechanical methods. They are applicable to large biomolecules and the first principles nature of the approach means it should be more applicable than fully empirical methods to partially unfolded conformations that may not be present in structural databases. Local excitation properties and exciton couplings can be computed with TDDFT. ${ }^{22}$ Much work has also been done on developing parameters from higher level multi-configurational ab initio methods for the relevant electronic excitations of the peptide backbone. ${ }^{11}$ These parameters and the computational methodology are available via the DichroCalc web interface ${ }^{23}$ and a github repository. ${ }^{14}$

Simulation of conformational dynamics and the associated CD spectra is proving to be a useful strategy for studying peptide and protein folding. A combination of CD spectroscopy, exciton calculations, standard MD simulations, and advanced-sampling methods allowed Hildebrand et al. ${ }^{24}$ to pinpoint a destabilization effect of silica on specific structural motifs of chymotrypsin. Using a similar strategy, Michaelis et al. ${ }^{25}$ demonstrated that an 
appropriate sampling of structures between a folded helix and its unfolded state permits one to generate reliable computed spectra of transient states. First and Webb ${ }^{26}$ performed MD folding simulations of a highly charged peptide in different solvents and bound to the surface of a methyl-terminated self-assembled monolayer. They found quantitative agreement between previous CD experiments and their MD simulations of solvent- and surface-induced conformational changes of the peptide. A related approach was used by Gattuso et al. ${ }^{27}$ to study the light-triggered conformational change of an $\alpha$-helical peptide of 27 amino acids with a retinal-like photoswitch covalently linked to the backbone. They correctly predicted the reduction in helicity of the peptide upon E to Z photoisomerisation of the switch.

Photoisomerisation of a molecular switch attached to the peptide backbone is strategy often employed to access fast timescales. ${ }^{28}$ Different molecular photo-switches have been studied. Of these, the azobenzene moiety has been frequently utilised. ${ }^{29,30}$ Azobenzene can be isomerised from the trans to cis and cis to trans conformation using a $370 \mathrm{~nm}$ and a visible light source $(460 \mathrm{~nm})$, respectively. ${ }^{31}$ Several groups have studied the dynamics of this photo-induced reaction and the isomerisation of the azobenzene appears to occur within one picosecond. ${ }^{32-35}$ The changes in the molecular geometry that occur over its isomerisation can be used to induce an imbalance in the structure of larger molecules, such as peptides and proteins. ${ }^{36-38}$

The study that we present here focuses on the azobenzene cross-linked 16 amino-acid peptide FK-11-X. ${ }^{29,39}$ The isomerisation of the azobenzene triggers the unfolding/folding process of the peptide. Experimental studies agree that, following the trans to cis azobenzene isomerisation, peptide conformational changes related to the unfolding process occur on the 100 ns to $1 \mu$ s time scale. ${ }^{40,41}$ Xia et al. ${ }^{42}$ used a combination of quantum mechanics/molecular mechanics $(\mathrm{QM} / \mathrm{MM})$ methods with a classical MM atomistic force field to simulate the unfolding process of a peptide with a similar sequence to FK-11-X. Although this approach successfully simulated the unfolding process of the peptide, the timescale of the dynamics did not match with the values found experimentally. In our study, using a 
purely classical MD approach, we sample significantly more of the conformational space.

In this study, we develop an atomistic force field for the azo-moiety of the FK-11-X peptide for use in MD simulations. We will describe the methodology we employed and the set of parameters we obtained. We utilise this atomistic force field to run $40 \mathrm{MD}$ trajectories simulating the unfolding process of FK-11-X. We characterise the folding state of the peptide over time using various structural properties and we compare the trajectories with each other. We calculate the rate of unfolding and compare this to experimental data. Using ensembles of computed molecular geometries, we calculate the time-evolution of the CD spectrum of FK-11-X as it unfolds. We connect these spectra to structural aspects of FK-11-X. Our results confirm that MD simulations can complement experimental measurements to give insights into transient conformations of small peptides as they unfold. Our assessment of the rate of the simulated unfolding process corresponds well to the value found experimentally, giving some confidence to the new atomistic force field parameters presented herein.

\section{Methods}

We built the peptide and the azo-moiety with the Avogadro software ${ }^{43}$ (version 1.2.0), using the feature that allows one to generate an $\alpha$-helix structure from an amino acid sequence. We constructed the azo-moiety in its trans configuration, placed it close to the position where it should be when it is linked to the peptide and created the bonds between the azo-moiety and the two sulfur atoms of the cysteines. The resulting structure after minimisation is shown in Figure 1. All the molecular visualisations presented in this paper were rendered using the Tachyon extension in VMD. ${ }^{44}$

We solvated the molecule using a box of TIP3P water. ${ }^{45}$ We determined the appropriate size of the water box from the length of the peptide and the cutoff distance of the nonbonded interaction we used for the simulation. We performed a preliminary MD simulation with the unfolded peptide to estimate of the maximum length of the peptide. The distance between the C-and N-termini never exceeded $35 \AA$. The cutoff distance for the non-bonded 
interactions is $12 \AA$, so we set the edge of the cubic water box at $50 \AA$. The solvated system comprises 15570 atoms.

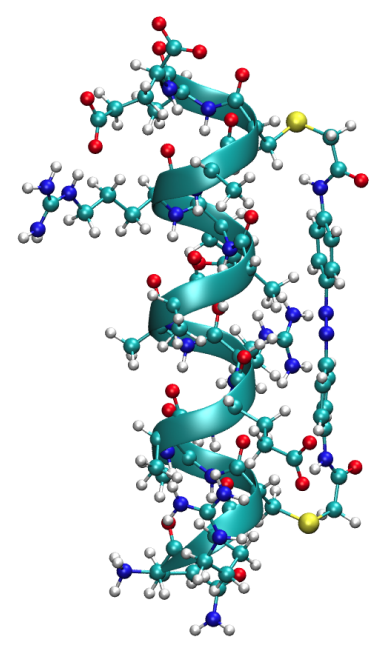

Figure 1: Structure of the FK-11-X peptide. Its amino acid sequence is Ac-Glu-Ala-Cys ${ }^{A Z O}$ Ala-Arg-Glu-Ala-Ala-Ala-Arg-Glu-Ala-Ala-Cys ${ }^{A Z O}$-Arg-Gln- $\mathrm{NH}_{2}$.

We performed the simulation using NAMD. ${ }^{46}$ It models the bonded and non-bonded interactions of each atom with all the other atoms of the system with a potential energy function whose form is well established in the field. We used the CHARMM36 All-Hydrogen force field ${ }^{47-49}$ to model the peptide. For the azo-moiety, Carstens ${ }^{50}$ developed parameters that have been used in some studies; ${ }^{39,40}$ and Böckmann et al. ${ }^{51}$ proposed a set of force field parameters for an azobenzene derivative. However, even if we could use these for the azobenzene, we would still need parameters for the atoms that link the peptide to the azobenzene. We used the online interface ${ }^{52}$ of the CHARMM General Force Field ${ }^{53}$ (CGenFF) to determine the required parameters by analogy with existing parameters of small organic molecules. This tool provides a penalty score for each attributed parameter. If this score is higher than 50, it is recommended to develop a customized force field for the corresponding bonds/angles. In the case of the azo-moiety, this score remains below 50 except for the parameters of dihedral angles. These dihedral angles have a relatively low contribution to the total potential energy. So we performed some preliminary tests using the CHARMM36 
a

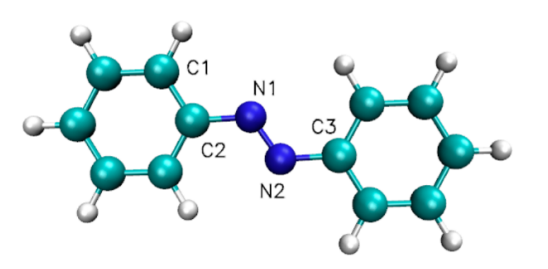

$\mathrm{b}$

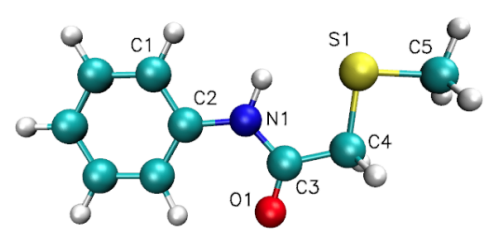

Figure 2: a. Azobenzene b. Structures used for the force field parameter optimisation of the linker between the azobenzene and the peptide.

The first step of the procedure is the partial atomic charge determination to attribute a charge to each atom. We generated the initial guesses with CGenFF. The penalty scores were below ten for all the atoms except for four atoms of the azobenzene $\left(\mathrm{C}_{2}, \mathrm{~N}_{1}, \mathrm{~N}_{2}\right.$ and 
$\mathrm{C}_{3}$ ). However, for these four atoms, differences between the charges generated by CGenFF and the charges proposed by Böckmann et al. ${ }^{51}$ were below $5 \%$. So we kept the initial guess of the partial atomic charges generated by CGenFF for the optimisation and for the simulations. We computed the QM geometries with Q-Chem ${ }^{55}$ at the $\mathrm{MP} 2 / 6-31 \mathrm{G}^{*}$ level of theory. We calculated the energy profile for all the bonds, the angles and the dihedral angles involving atoms that are indexed in Figure 2. The parameters of the benzene rings and the parameters of the bonds/angles involving hydrogens are generated by CGenFF.

We performed the MM minimisation of the structures using NAMD. We adjusted the parameters 'by hand' until the energy profiles from MM fit with the profile from QM. We first optimised the parameters of the bonds, then the parameters of the angles and finally the parameters of the dihedral angles. Examples of the potential energy surfaces are shown in Figure 3. Both the MM geometries computed with initial guess and optimised parameters are represented. The energy profiles calculated with the initial guess from CGenFF are significantly different compared to the profiles based on QM calculations. These differences may be responsible for the qualitatively incorrect behaviour we observed in the preliminary simulations using the initial guess. The profiles obtained with the optimised parameters are closer to the target and should improve the fidelity of the simulation. The optimised parameters are presented in Tables 1 and 2, and are compared in the Supplementary Information (Tables S1 and S2).

The MD simulations were performed with NAMD 2.12 with a 2 fs timestep. The temperature was maintained at $295 \mathrm{~K}$ using Langevin dynamics parameters with a friction coefficient of $5 \mathrm{ps}^{-1}$. The pressure was kept constant at 1 atm with a Langevin piston that modifies the size of the unit cell to adjust the pressure. The maximum distance of non-bonded interactions was $12 \AA$. We used cubic periodic boundary conditions. Electrostatics were treated using the particle-mesh Ewald method ${ }^{56}$ with a grid spacing of $1 \AA$. We used the SETTLE $\operatorname{algorithm~}^{57}$ and the ShakeH algorithm ${ }^{58}$ to constrain water molecules and the other bonds involving hydrogen atoms, respectively. We performed two sets of simulations. The first 
is composed of 40 trajectories where the azobenzene is isomerised in the cis form and a second set of 10 trajectories with the trans configuration of the azobenzene, which served as a control. Each of the trajectories consisted of 120 ns of production dynamics. In each case, the initial structure was minimized for 200 steps and was equilibrated for 200 ps, which was sufficient based on the convergence of the RMSD (Supplementary Information, Figure S1).
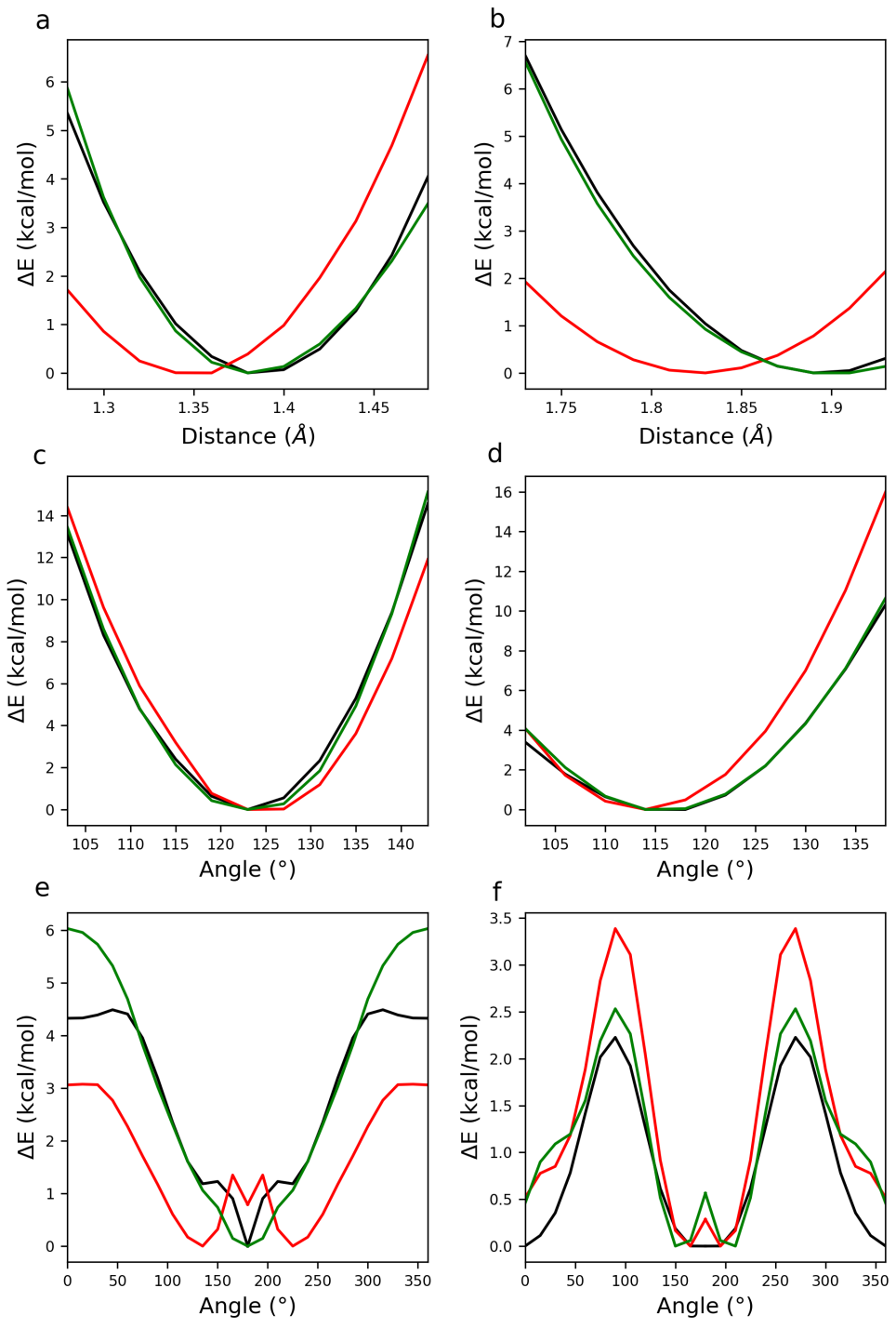

Figure 3: Examples of potential energy profiles of the structure shown in Figure 2b. The profile from MP2/6-31G* geometries are plotted in black. The profile from MM geometries minimised using initial guess and optimised parameters are plotted in red and green, respectively. a. $\mathrm{N}_{1} \mathrm{C}_{3}$ bond b. $\mathrm{C}_{4} \mathrm{~S}_{1}$ bond c. $\mathrm{C}_{1} \mathrm{C}_{2} \mathrm{~N}_{1}$ angle d. $\mathrm{C}_{3} \mathrm{C}_{4} \mathrm{~S}_{1}$ angle e. $\mathrm{O}_{1} \mathrm{C}_{3} \mathrm{C}_{4} \mathrm{~S}_{1}$ dihedral angle f. $\mathrm{C}_{1} \mathrm{C}_{2} \mathrm{~N}_{1} \mathrm{C}_{3}$ dihedral angle. 
Table 1: Optimised force field parameters of the azobenzene (Figure 2a).

\begin{tabular}{lccc}
\hline Dihedral angle & $k(\mathrm{kcal} / \mathrm{mol})$ & $n$ & $\delta\left(^{\circ}\right)$ \\
\hline $\mathrm{C}_{1} \mathrm{C}_{2} \mathrm{~N}_{1} \mathrm{~N}_{2}$ & 2.5 & 2 & 180 \\
$\mathrm{C}_{2} \mathrm{~N}_{1} \mathrm{~N}_{2} \mathrm{C}_{3}$ & 23.0 & 2 & 180 \\
\hline
\end{tabular}

\begin{tabular}{lcc}
\hline Angle & $k(\mathrm{kcal} / \mathrm{mol})$ & $\theta_{0}\left(^{\circ}\right)$ \\
\hline $\mathrm{C}_{1} \mathrm{C}_{2} \mathrm{~N}_{1}$ & 50 & 116.5 \\
& 75 & 106.0
\end{tabular}

\begin{tabular}{lcc}
\hline Bond & $k(\mathrm{kcal} / \mathrm{mol})$ & $b_{0}(\AA)$ \\
\hline $\mathrm{C}_{2} \mathrm{~N}_{1}$ & 300 & 1.415
\end{tabular}

Table 2: Optimised force field parameters of the molecule shown in Figure 2b.

\begin{tabular}{cccc}
\hline Dihedral angle & $k(\mathrm{kcal} / \mathrm{mol})$ & $n$ & $\delta\left(^{\circ}\right)$ \\
\hline $\mathrm{C}_{1} \mathrm{C}_{2} \mathrm{~N}_{1} \mathrm{C}_{3}$ & 1.20 & 2 & 180 \\
$\mathrm{C}_{2} \mathrm{~N}_{1} \mathrm{C}_{3} \mathrm{O}_{1}$ & 2.65 & 2 & 180 \\
$\mathrm{C}_{2} \mathrm{~N}_{1} \mathrm{C}_{3} \mathrm{C}_{4}$ & 2.00 & 2 & 180 \\
$\mathrm{~N}_{1} \mathrm{C}_{3} \mathrm{C}_{4} \mathrm{~S}_{1}$ & 0.50 & 1 & 180 \\
$\mathrm{O}_{1} \mathrm{C}_{3} \mathrm{C}_{4} \mathrm{~S}_{1}$ & 0.50 & 1 & 0 \\
$\mathrm{C}_{3} \mathrm{C}_{4} \mathrm{~S}_{1} \mathrm{C}_{5}$ & 0.24 & 1 & 180 \\
\hline
\end{tabular}

\begin{tabular}{ccc}
\hline Angle & $k(\mathrm{kcal} / \mathrm{mol})$ & $\theta_{0}\left(^{\circ}\right)$ \\
\hline $\mathrm{C}_{1} \mathrm{C}_{2} \mathrm{~N}_{1}$ & 70 & 115.2 \\
$\mathrm{C}_{2} \mathrm{~N}_{1} \mathrm{C}_{3}$ & 60 & 120.8 \\
$\mathrm{~N}_{1} \mathrm{C}_{3} \mathrm{O}_{1}$ & 92 & 124.5 \\
$\mathrm{~N}_{1} \mathrm{C}_{3} \mathrm{C}_{4}$ & 68 & 118.0 \\
$\mathrm{O}_{1} \mathrm{C}_{3} \mathrm{C}_{4}$ & 78 & 122.0 \\
$\mathrm{C}_{3} \mathrm{C}_{4} \mathrm{~S}_{1}$ & 40 & 115.3 \\
$\mathrm{C}_{4} \mathrm{~S}_{1} \mathrm{C}_{5}$ & 58 & 99.0 \\
\hline
\end{tabular}

\begin{tabular}{lcc}
\hline Bond & $k(\mathrm{kcal} / \mathrm{mol})$ & $b_{0}(\AA)$ \\
\hline $\mathrm{C}_{2} \mathrm{~N}_{1}$ & 380 & 1.420 \\
$\mathrm{~N}_{1} \mathrm{C}_{3}$ & 460 & 1.385 \\
$\mathrm{C}_{3} \mathrm{O}_{1}$ & 830 & 1.275 \\
$\mathrm{C}_{3} \mathrm{C}_{4}$ & 250 & 1.542 \\
$\mathrm{C}_{4} \mathrm{~S}_{1}$ & 225 & 1.893 \\
$\mathrm{~S}_{1} \mathrm{C}_{5}$ & 225 & 1.893 \\
\hline
\end{tabular}

There are two methods to trigger the isomerisation of the azo-moiety. One can use a QM approach ${ }^{42}$ or a potential energy triggering. ${ }^{59}$ We used the second option. The principle of this method is to change the parameters of the dihedral angle responsible of the azobenzene configuration (C-N-N-C). We modified the multiplicity of this dihedral from two to one (see Figure 4a). This modification changes the number of minima from two to one. The unique energy minimum is located at $0^{\circ}$, which corresponds to the cis configuration of the azobenzene. We performed a $1.5 \mathrm{ps}$ simulation with this modification for each structure starting from the conformation of the last step from the equilibration trajectory. The averaged value of the C-N-N-C angle of the azobenzene over time is plotted in Figure 4b. We see that the dihedral angle decreases from $180^{\circ}$ to approximately $60^{\circ}$ before the first picosecond. The angle passes the potential barriers located at $-90^{\circ}$ and $+90^{\circ}$ when $n=2$ and so the azobenzene will remain in the cis conformation even with $n=2$. We used the conformation of the last step from the 40 isomerisation trajectories for the 120 ns simulations. For these simulations, the multiplicity parameter of the C-N-N-C dihedral angle was $n=2$. 
a

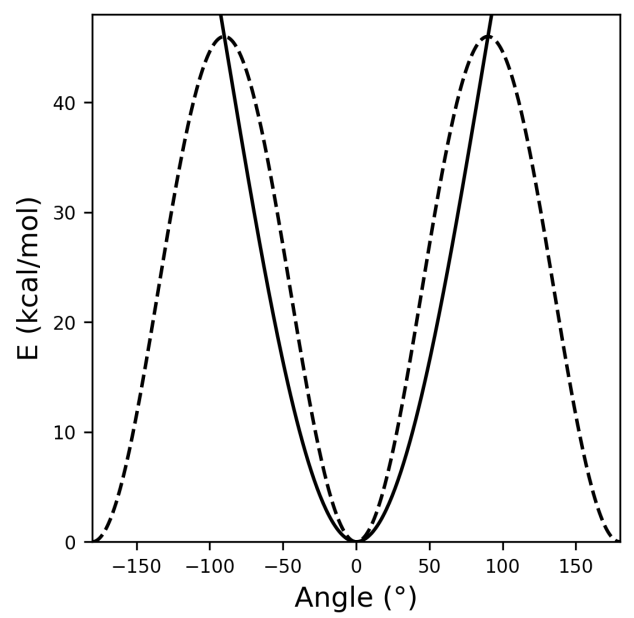

b

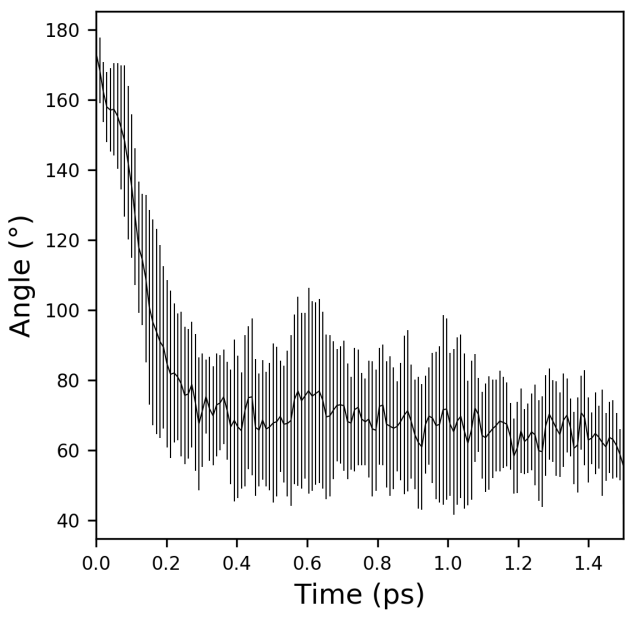

Figure 4: a. C-N-N-C dihedral angle energy function with multiplicity $n=1$ (solid line) and $n=2$ (dashed line). b. Averaged $\mathrm{C}-\mathrm{N}-\mathrm{N}-\mathrm{C}$ dihedral angle value over the isomerisation trajectories. The error bars correspond to one standard deviation.

Theoretical CD spectra were calculated using the DichroCalc web interface ${ }^{23}$ for individual frames of the simulated trajectories. DichroCalc constructs an exciton Hamiltonian matrix using electronic excitations of the local peptide chromophores. The Hamiltonian models how electronic transitions interact with one another. The sign and magnitude of the interactions depend on the relative orientation and separation of chromophores, which in turn arise from the precise structure of the peptide. For each peptide chromophore two transitions, $n \rightarrow \pi^{*}$ and $\pi_{n b} \rightarrow \pi^{*}$, were considered using an ab initio parameter set. ${ }^{11}$ Other transitions, such as the $\pi_{b} \rightarrow \pi^{*}$ and charge transfer transitions, were not included. The calculated line spectra were convoluted with Gaussian bands of bandwidth $12.5 \mathrm{~nm}$.

\section{Results \& Discussion}

The trajectories are, on the whole, relatively similar to one another. The similarity amongst the trajectories was assessed by computing the root mean squared inner product between pairs of trajectories, ${ }^{60}$ where each trajectory was represented by the first principal component of the backbone dynamics computed over the final 5 ns. The tenth trajectory appears to be the most representative, as it has the highest average RMSIP value (0.51) over the 39 
pairwise comparisons that include the tenth trajectory. We performed this analysis with the Python package ProDy. ${ }^{61}$ Five snapshots of the tenth trajectory are shown in Figure 5. The peptide starts to unfold within the first $6 \mathrm{~ns}$ and keeps the same partially unfolded structure after $40 \mathrm{~ns}$, approximately. Thus, the peptide eventually attains this conformation, which seems stable, in most trajectories. The helical structure that remains is formed by six residues (Ala-Arg-Glu-Ala-Ala-Ala). The three consecutive alanines are localised at the centre of the peptide. Alanine has a propensity to form and stabilise $\alpha$-helix structure. ${ }^{62}$ This property and central location likely explain the high stability of this part of the peptide $\alpha$-helix conformation.

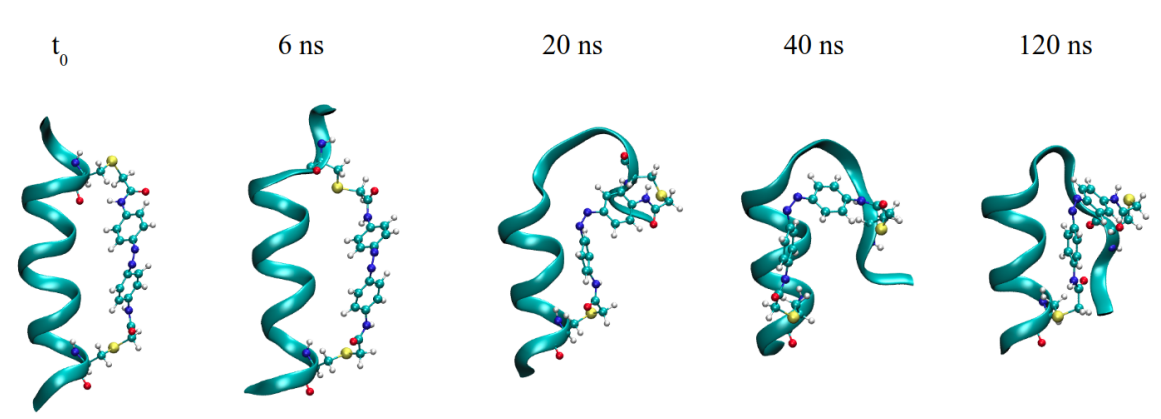

Figure 5: Typical trajectory from the 40 simulations.

We also identified other trajectories where the unfolding process is in a more advanced state after $120 \mathrm{~ns}$. One of these trajectories is shown in Figure 6. In this case, the peptide is completely unfolded after 80 ns. Although the structure of the $\alpha$-helix is modified after $80 \mathrm{ps}$, it only starts to unfold after $200 \mathrm{ps}$. The conformation at $10 \mathrm{~ns}$ is similar to the stable conformation we identified in Figure 5. However, in this case, the peptide continues to unfold and the unfolding process is completed after $80 \mathrm{~ns}$, approximately. The trajectory in Figure 6 is less frequent than the type shown in Figure 5. It occurs only once in the set of 40 trajectories. We can gather more detailed information about the unfolding process using a Ramachandran plot representation (Figure 7) showing each residue from the trajectory presented in Figure 6.

Figure 7 allows us to obtain information about the secondary structure of each residue. ${ }^{63}$ 
The two most represented secondary structures are the $\alpha$-helix and the $\beta$-strand. They correspond to the cluster of points centered around $\phi=-57^{\circ}, \psi=-47^{\circ}$ and $\phi=-80^{\circ}$, $\psi=150^{\circ}$, respectively. As expected, all the residues form an $\alpha$-helix structure at the beginning of the simulation. The dihedral angles of the residues Arg2, Glu6, Ala8 and Ala15 are the first to change. They are followed by the residues Ala9, Ala10, Arg12 and Cys14, which switch from the helical region to the extended structure region of the Ramachandran plot. The dihedral angles of the residues Arg2, Cys3, Ala8 and Ala15 return approximately to their initial value at the end of the simulation. We also observe that the dihedral angles of the residues Arg2, Ala9, Ala10, and Arg12 transiently populate the upper-right quadrant of the Ramachandran plot, which corresponds to the region of the left-handed $\alpha$-helix structure.

In order to quantify the rate of the unfolding process we examine some representative quantitative progress coordinates, such as the distance between the two sulfur atoms and the $\mathrm{O}_{i}-\mathrm{H}_{i+4}$ distances. The S-S distance is directly related to the structure of the azo-moiety. 

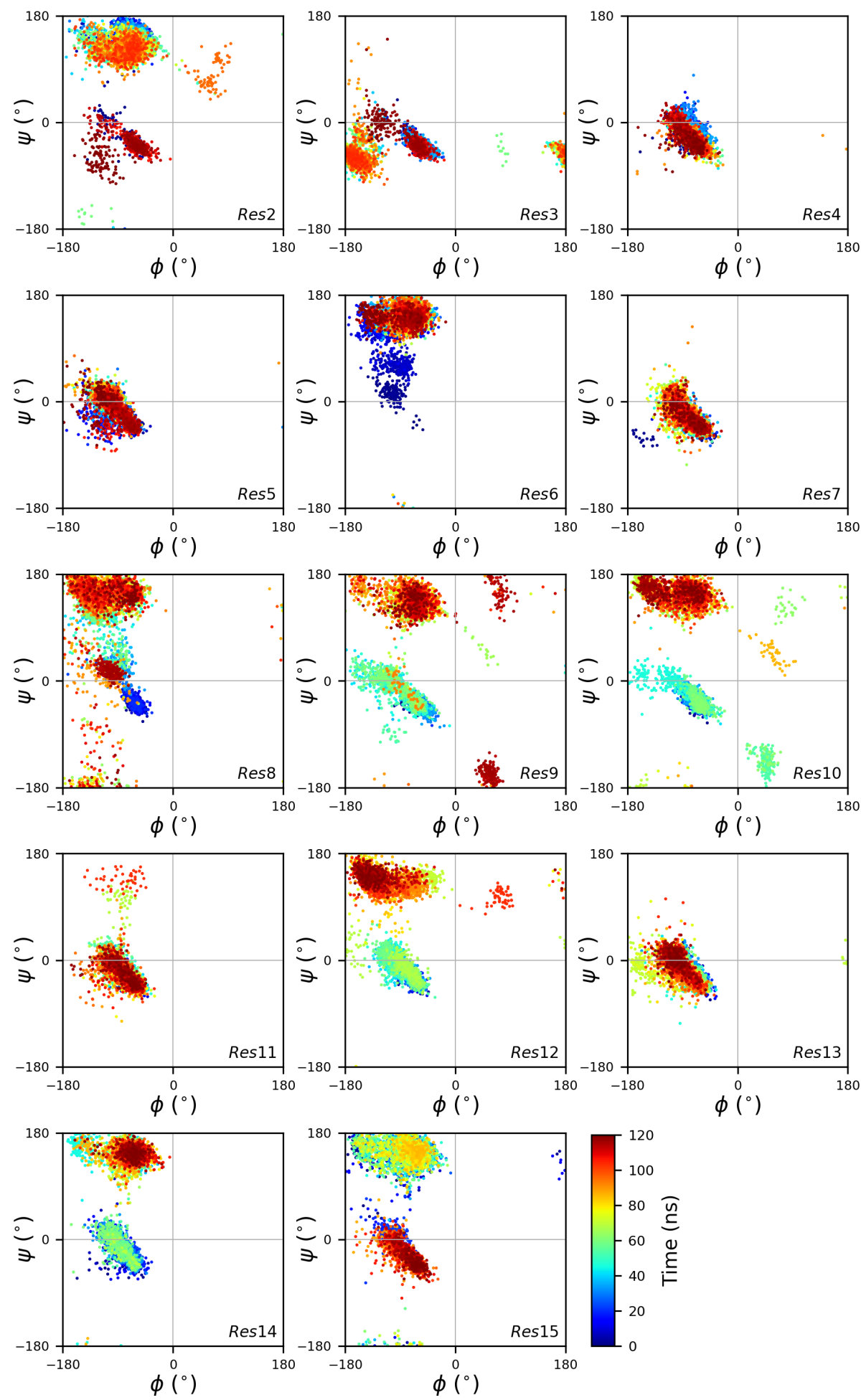

Figure 7: Ramachandran plots of the trajectory shown in Figure 6. The colour of the scatter plot corresponds to the time of the simulation. 
We expect that, once isomerised, the azo-moiety tends to a lower energy conformation with this configuration. We also think that as long as the distance between the two sulfur atoms decreases, the azo-moiety induces a strain on the peptide. The hydrogen bond between $\mathrm{O}_{i}$ and $\mathrm{H}_{i+4}$ characterises the $\alpha$-helix structure and the distance between these two atoms allows one to assess the stability of the structure. ${ }^{64}$ The averaged $\mathrm{O}_{i}-\mathrm{H}_{i+4}$ distance of the peptide residues will probably be partially correlated to the distance between the two sulfur atoms but we can expect that the conformation of the peptide continues to change even after the S-S distance converges. The S-S distance and the $\mathrm{O}_{i}-\mathrm{H}_{i+4}$ distances are presented in Figures 8 and 9 , respectively.

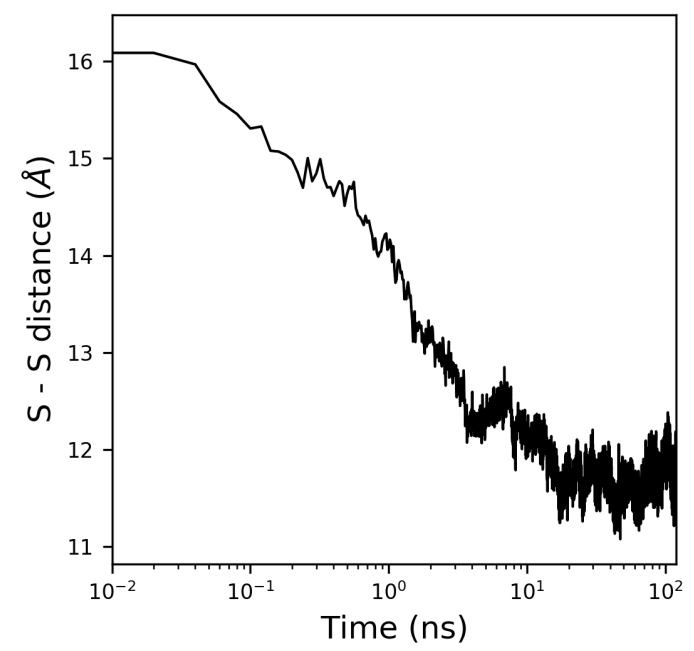

Figure 8: Distance between the two sulfur atoms of the cysteines over time (logarithmic scale).

In Figure 8, the S-S distance decreases from $16 \AA$ to $12 \AA$ within the first 10 ns of the simulation. Then it stabilises between $11 \AA$ and $12 \AA$ until the end of the simulation. We see in Figure 9a the heterogeneity in the speed of unfolding over the 40 trajectories. The trajectory in Figure 6 is trajectory number 26. Its mean $\mathrm{O}_{i}-\mathrm{H}_{i+4}$ distance (averaged over residues) is plotted against time in Figure $9 \mathrm{~b}$. The averaged $\mathrm{O}_{i}-\mathrm{H}_{i+4}$ distance from the 40 trajectories with the cis azo-moiety is shown in Figure 9c. The abscissa scale is logarithmic so the $\mathrm{O}_{i}-\mathrm{H}_{i+4}$ distance increases exponentially. The averaged $\mathrm{O}_{i}-\mathrm{H}_{i+4}$ distance increases 

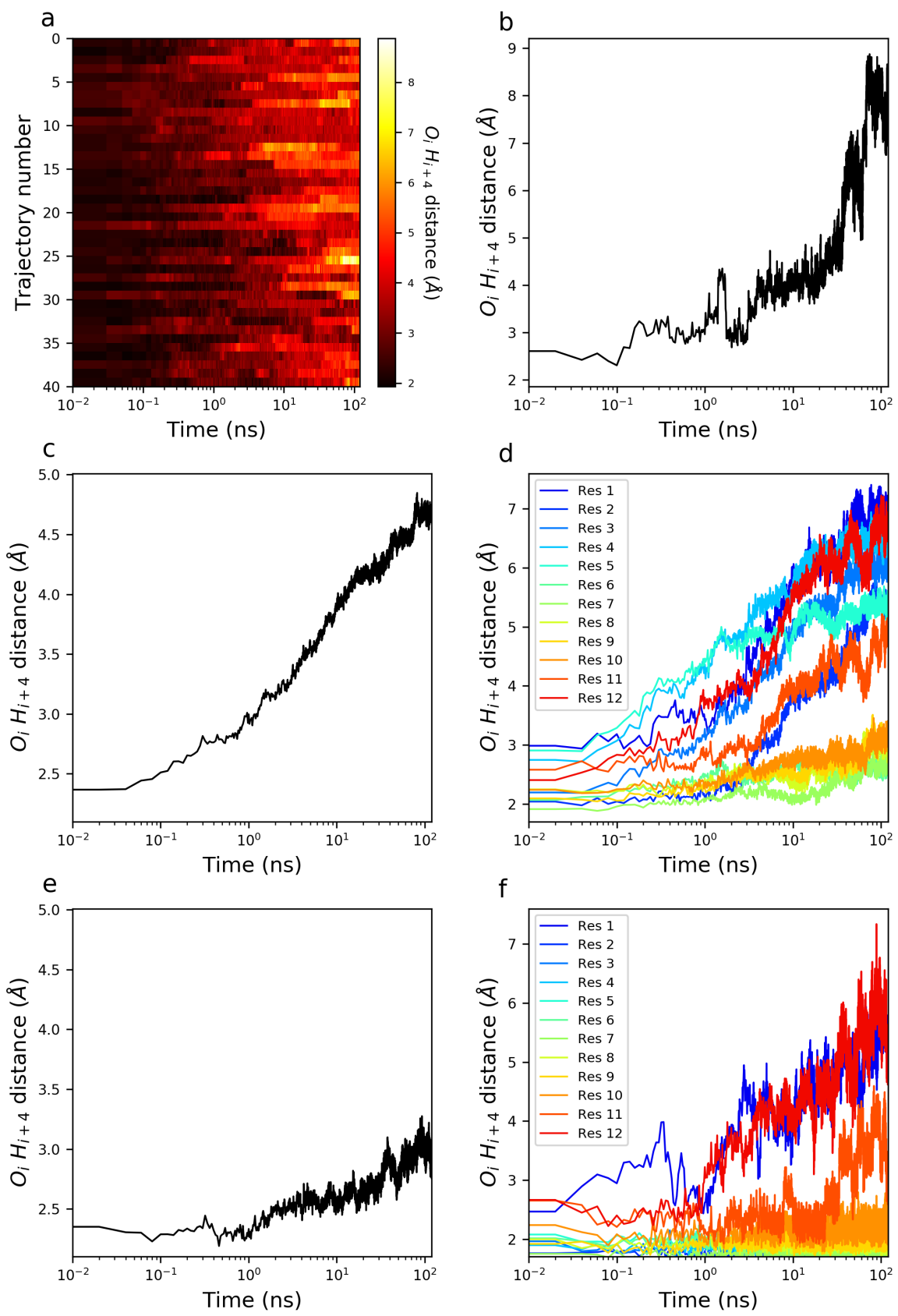

Figure 9: a. 2D representation of the averaged $\mathrm{O}_{i}-\mathrm{H}_{i+4}$ distance for each trajectory over time. b. Averaged $\mathrm{O}_{i^{-}} \mathrm{H}_{i+4}$ distance of the trajectory shown in Figure 6 over time. c. Averaged $\mathrm{O}_{i^{-}}$ $\mathrm{H}_{i+4}$ distance of the 40 trajectories with the cis azo-moiety over time. d. Averaged $\mathrm{O}_{i}-\mathrm{H}_{i+4}$ distance of the 40 trajectories with the cis azo-moiety per residue over time. e. Averaged $\mathrm{O}_{i}-\mathrm{H}_{i+4}$ distance of the 10 trajectories with the trans azo-moiety over time. f. Averaged $\mathrm{O}_{i}-\mathrm{H}_{i+4}$ distance of the 10 trajectories with the trans azo-moiety per residue over time. 
after 10 ns even though the S-S distance is constant. Thus, the peptide structure continues to change. In Figure $9 \mathrm{~b}$ we see that the averaged $\mathrm{O}_{i}-\mathrm{H}_{i+4}$ distance when the peptide is completely unfolded is approximately $7.5 \AA$. Considering that Figure $9 \mathrm{c}$ shows that the averaged $\mathrm{O}_{i}-\mathrm{H}_{i+4}$ distance increases by approximately $1 \AA$ every order of magnitude and reaches 4.5 $\AA$ after 100 ns, it suggests that all the peptides should be completely unfolded after 100 $\mu \mathrm{s}$. This extrapolated value does not correspond to values determined experimentally, which range between $100 \mathrm{~ns}$ and $1 \mu \mathrm{s} .{ }^{40,41}$ We will continue this comparison with experimental measurements in the next section. In Figure $9 \mathrm{~d}$, we plot the averaged $\mathrm{O}_{i}-\mathrm{H}_{i+4}$ distance for the first 12 residues. This representation confirms that the $\alpha$-helix structure of the residues located at the centre of the peptide is more stable than at its termini. Earlier, we presented the hypothesis that the FK-11-X reaches a stable conformation after 40 ns approximately. We see in Figure 9d that the $\mathrm{O}_{i}-\mathrm{H}_{i+4}$ distances of the residues Ala10 to Glu6 continue to increase even after 40 ns. It could mean that the stable conformation shown in Figure 5 might be a local minimum and that the peptide will finally unfold completely on a longer time scale. The control simulation is shown in Figures 9e and 9f, which show the averaged $\mathrm{O}_{i}-\mathrm{H}_{i+4}$ distances and the averaged $\mathrm{O}_{i}-\mathrm{H}_{i+4}$ distances, respectively, per residue from the ten trajectories with the trans azo-moiety. These two figures show that the termini of the $\alpha$-helix structure are not stable on the nanosecond time scale. However, we see a clear difference between the central residues with the cis and with the trans azo-moiety, which confirm the impact of the isomerisation of the azo-moiety on the peptide structure.

We performed an analysis based on CD spectra computed with the online version of Dichrocalc. ${ }^{23}$ We calculated the CD spectra of the structures from MD simulations at 11 different times $(0,0.02,0.08,0.2,0.8,2,8,20,30,60$, and $120 \mathrm{~ns})$. We selected these time points to sample homogeneously an exponential decay. The spectra of the trajectory illustrated in Figure 6 are shown in Figure 10. The CD spectrum at $t_{0}$ is close to the spectral signature of an $\alpha$-helix with a positive peak at $190 \mathrm{~nm}$ and a negative peak between 200 and $230 \mathrm{~nm}$, centered at $208 \mathrm{~nm}$. Whilst the well-known band at $222 \mathrm{~nm}$ appears as a shoulder 


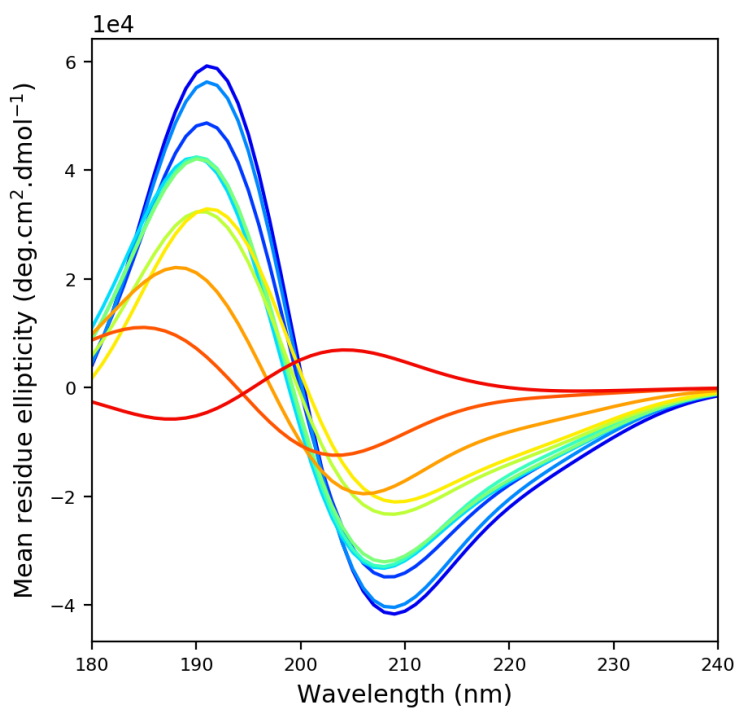

Figure 10: CD spectra calculated from the trajectory shown in Figure 6. The spectra belong to the structures extracted at 0 (blue), 0.02, 0.08, 0.2, 0.8, 2, 8, 20,30, 60, and $120 \mathrm{~ns}$ (red).

rather than being more clearly distinct from the $208 \mathrm{~nm}$ band, the calculated intensity at $222 \mathrm{~nm}$ has been previously shown to be highly correlated with the experimental intensity at that wavelength. ${ }^{11}$ The amplitude of the signal decreases progressively over the simulation. We also noticed that the spectrum of the last time frame, at $120 \mathrm{~ns}$, is different. It exhibits a negative peak at $190 \mathrm{~nm}$ and a positive peak at $205 \mathrm{~nm}$. This spectrum corresponds to the spectral signature of a random coil. Figure 6 shows that the $\alpha$-helix content of the peptide decreases over the simulation and it is completely unfolded after $80 \mathrm{~ns}$. In the literature, the stable conformation of the cis FK-11-X peptide has been associated with the conformation at $120 \mathrm{~ns}$ in Figure $6{ }^{39}$ The experimental synchrotron radiation CD (SRCD) spectra of trans and the cis configuration of FK-11-X have the same shape but with different amplitudes ${ }^{65}$ (Figure 11b). Thus, the stable conformation of the cis FK-11-X is partially helical. If it were not, its CD spectra would correspond to a random coil based structure as the computed CD spectra at 120 ns (Figure 10). In addition, Auvray et al. ${ }^{65}$ recently showed that the use of the natural polarisation of the synchrotron radiation may improve the temporal resolution of the technique to reach sub-nanosecond resolution. They assessed 

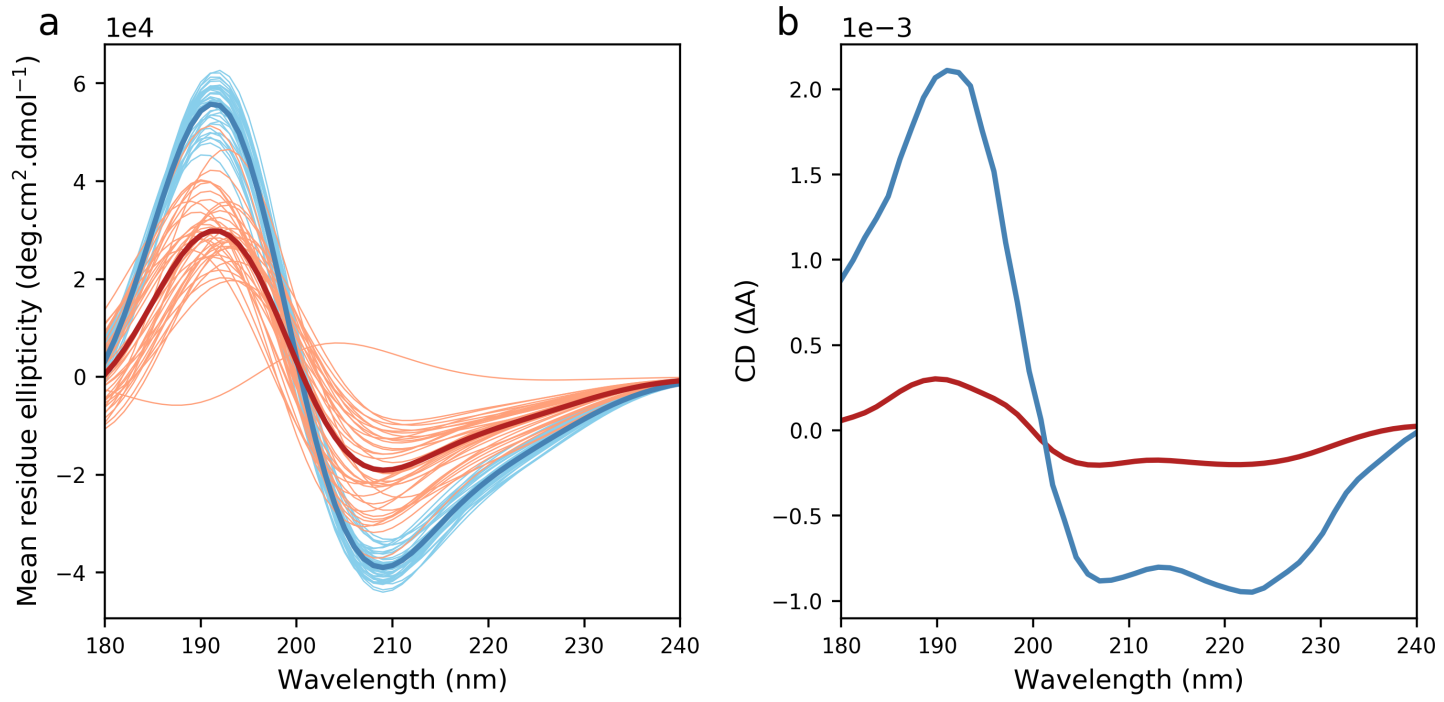

Figure 11: a. The computed CD spectra of the 40 trajectories from structures extracted at $t=0 \mathrm{~ns}$ (blue) and $t=120 \mathrm{~ns}$ (red). The thicker curves correspond to the average spectra. b. SRCD spectra of cis (red) and trans (blue) FK-11-X, as published in ref [63] under a Creative Commons CC BY license.

SRCD spectra plotted in Figure 11b show that the amplitude of the CD signal at $222 \mathrm{~nm}$ decreases by $80 \%$ when the configuration of the azo-moiety passes from trans to cis. The 
same decrease in amplitude of the averaged CD spectrum computed with the structures at $t=0 \mathrm{~ns}$ (trans configuration) would result in a computed CD spectrum with an amplitude of $-3,600 \mathrm{deg} \mathrm{cm}^{2} \mathrm{dmol}^{-1}$ at $222 \mathrm{~nm}$. The amplitude of the average of the CD spectra computed with the structures at $t=120 \mathrm{~ns}$ (cis configuration) is $-9,800 \mathrm{deg} \mathrm{cm}^{2} \mathrm{dmol}^{-1}$ at $222 \mathrm{~nm}$. Thus, it decreases by $46 \%$ within the first 120 ns of simulation. The differences may come from either the sampling and could be addressed by increasing the number of trajectories or the duration of the simulation. We observed in Figure $9 \mathrm{~b}$ that the averaged $\mathrm{O}_{i}-\mathrm{H}_{i+4}$ distance continues to increase at the end of the simulation, so we can expect that the average helical content of the 40 trajectories tends to the value determined experimentally within the next tens of nanoseconds.

In Figure 11a we see that in some trajectories, the peptide is in a more advanced unfolding state, in which the $\mathrm{CD}$ at $222 \mathrm{~nm}$ decreases to $-5,000 \mathrm{deg} \mathrm{cm}^{2} \mathrm{dmol}^{-1}$, approximately, which corresponds to a decrease of $70 \%$. This estimation is close to the value determined with experimental results and thus the computed structure of the peptide at 120 ns in these trajectories may correspond to the most likely conformation of the FK-11-X in the cis configuration. The structures from three of these trajectories are shown in Figure 12. The helical content that remains in these structures is formed by the same residues as the helix shown in Figure 5, which include four alanines (Ala-Arg-Glu-Ala-Ala-Ala). Again, it shows the propensity of alanines to stabilise $\alpha$-helix structure.

Chen et al. ${ }^{40}$ used data from time-resolved ORD experiments to estimate a time constant of $55 \pm 6$ ns (single-exponential decay) for the unfolding process of the FK-11-X peptide. This estimation is based on data points measured from 50 ns to $10 \mu$ s after the trigger of the isomerisation. The lack of information over the first $50 \mathrm{~ns}$ leads to a significant overestimation of the value of the time constant. Based on this time-constant estimation, we can deduce that the dynamics should reach $95 \%$ of its total variation after $165 \pm 6$ ns. So, even if this estimation may be too long, it shows that the unfolding process occurs within the first $165 \pm 6$ ns after the isomerisation of the azobenzene. In order to estimate the rate of the 


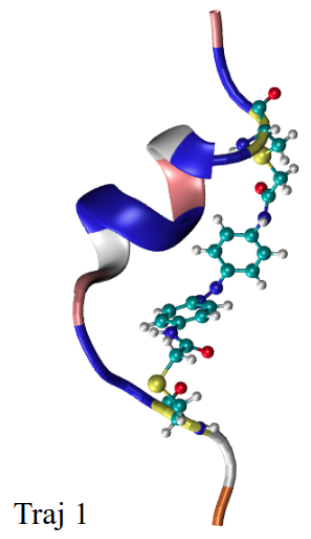

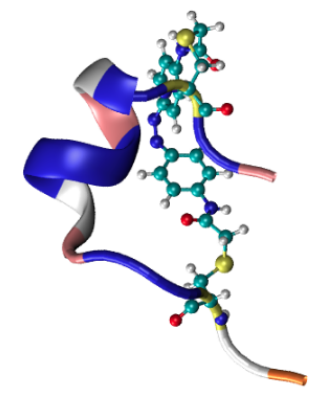

Traj 20

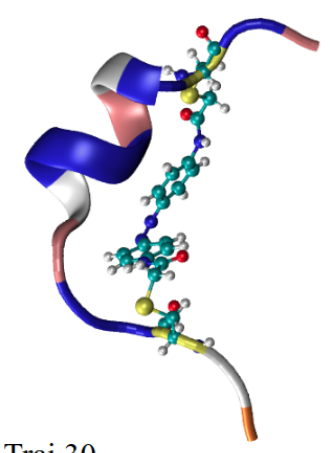

Traj 30

Figure 12: Structure of the FK-11-X peptide at 120 ns of the trajectories 1, 20 and 30. The colors of the peptide correspond to the amino acid type. Gln, Arg, Cys, Ala and Glu are in orange, white, yellow, blue and pink, respectively.

unfolding process via the change in CD, we averaged the computed CD spectra from the 40 simulations at $0,0.02,0.08,0.2,0.8,2,8,20,30,60$, and 120 ns. The averaged computed CD spectra and the trace of the CD signal at $222 \mathrm{~nm}$ are plotted in Figures 13a and 13b. The average spectra show that the amplitude of the CD signal decreases continuously over the simulation and homogeneously between 180 and $240 \mathrm{~nm}$. The time-evolution of the intensity at $222 \mathrm{~nm}$ (Figure 13b) is sigmoidal, albeit not sharply so, reflecting a cooperative process, which evident in the correlated increase of several of the $\mathrm{O}_{i}-\mathrm{H}_{i+4}$ distances seen in Figure $9 \mathrm{~d}$. In Figure 13b, the signal decreases rapidly over the first 20 ns and it starts to stabilise after $20 \mathrm{~ns}$. So we can expect that the signal continues to decrease slightly over the next tens of nanoseconds. We performed a non-weighted least-squares fitting with a single-exponential decay model of the computed CD signal at $222 \mathrm{~nm}$. The resulting curve is plotted in Figure 13b. This fitting allows us to estimate the time-constant of the simulated unfolding process at $\tau=5.80 \pm 0.03$ ns. Because we see that the computed CD signal seems to not reach a plateau at the end of the simulation, this may be an under-estimate and could be refined with longer simulations or perhaps with simulations using a polarisable force field. The final slower decrease in amplitude expected after 120 ns should make the loss of amplitude at 222 $\mathrm{nm}$ approach $80 \%$, the value that we deduced from experimental data. These results show 

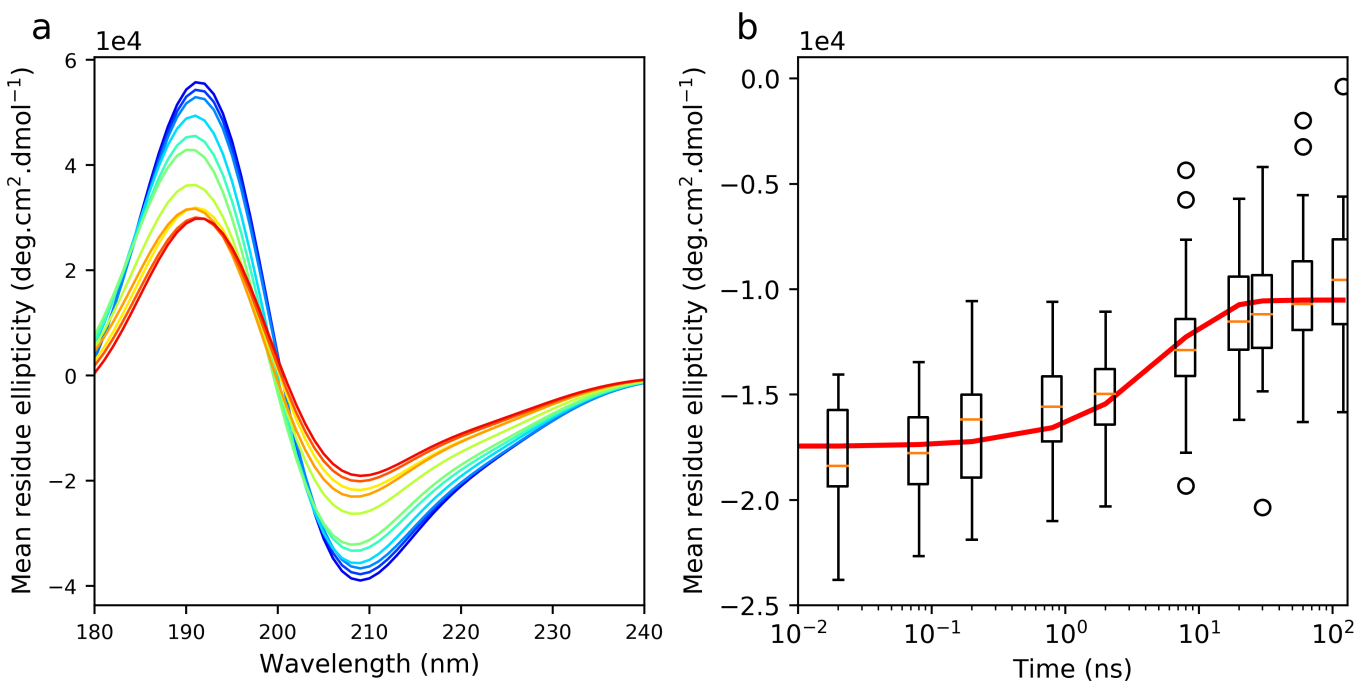

Figure 13: a. Averaged CD spectra of the 40 trajectories from structures extracted at 0 (blue), 0.02, 0.08, 0.2, 0.8, 2, 8, 20, 30, 60, and $120 \mathrm{~ns}$ (red). b. Trace of the computed CD signal at $222 \mathrm{~nm}$. The boxes extend from the first to the third quartile values of the data, with a horizontal red line at the median. The whiskers attached to the boxes extend to the extrema of the data. If the whiskers are $50 \%$ longer than the difference between the first and the third quartile values, then the outer data points are represented as circles. The red curve is a non-weighted least-squares fitting with a single-exponential decay model with a time constant of $\tau=5.80 \pm 0.03$ ns.

that the rate of the simulated unfolding process is consistent with that provided by timeresolved ORD experiments. Comparison with the time-resolved ORD experiment of Chen et $a l .{ }^{40}$ is more relevant than comparison with the time-resolved IR data from Bredenbeck et al., ${ }^{41}$ because the trans to cis isomerization is followed by equilibration on the cis energy landscape, whereas the latter investigated dynamics on the trans landscape.

Khuc et al. ${ }^{6}$ performed quantitative time-resolved CD measurement with a time step of $12 \mathrm{~ns}$ at $225 \mathrm{~nm}$. They successfully estimated the variation of the helical fraction of a poly(glutamic acid) peptide over a T-jump experiment using a nanosecond Nd:YAG laser to heat up the solvent and a sub-picosecond source based on a $1 \mathrm{kHz}$ amplified TitaniumSapphire system for the CD measurement. ${ }^{66}$ Dartigalongue et al. ${ }^{66}$ used this light source to perfom sub-picosecond measurements to assess the change in CD over the photodissociation of carbonmonoxy-myoglobin. Thus, time-resolved pump-probe CD measurements with either sub-picosecond or nanosecond temporal resolution on the FK-11-X peptide using 


\section{Conclusion}

We have investigated the unfolding of the FK-11-X peptide based on a computational approach. We optimised a force field for the azo-moiety to ensure that this part of the FK-11-X peptide is parameterised well and in an internally consistent fashion. Our parameters should find utility in future simulation studies of other azo-peptides. Using this force field, we computed $40 \mathrm{MD}$ trajectories of $120 \mathrm{~ns}$ simulating the unfolding process of the FK-11-X peptide. Approximately $80 \%$ of the trajectories presented a partially unfolded peptide with a similar conformation at the end of the simulation. The remaining $20 \%$ were in a more advanced unfolded state and in one trajectory the peptide was completely unfolded. We analysed the dynamics of the process looking at the distance between the two sulfur atoms and the $\mathrm{O}_{i}-\mathrm{H}_{i+4}$ backbone hydrogen bond distances. The S-S distance decreases progressively during the first $10 \mathrm{~ns}$ and then is stable until the end of the simulation. The $\mathrm{O}_{i}-\mathrm{H}_{i+4}$ distances and the structure of the peptide at 120 ns indicated that the three alanines located between the two cysteines significantly stabilise the $\alpha$-helix. We also see that the termini of the FK-11-X helix are quite labile on the nanosecond timescale, even in the trans configuration. The amino acids at the termini unfold progressively over the first 100 ns. An analysis of CD spectra computed from snapshots from the MD simulations allowed us to compare the dynamics of the simulated unfolding process and the experimental data, with the caveat that an improved model of the coupling of the $\pi \pi^{*}$ and $n \pi^{*}$ transitions might resolve the calculated band at $222 \mathrm{~nm}$ better. The calculated CD signal at $222 \mathrm{~nm}$ reaches a plateau at the end of the simulation. The $55 \pm 6$ ns time constant proposed by Chen et al. allows us to estimate that $95 \%$ of the reaction would occur within $165 \pm 6$ ns. This estimate matches well with the rate of the simulated dynamics. We also used the variation of the CD signal at $222 \mathrm{~nm}$ from experimental data and from the calculated spectra to demonstrate that the 
most likely stable conformation of the cis FK-11-X is not the fully unfolded conformation but a conformation close to the structure shown in Figure 12.

\section{Supporting Information Available}

The supporting information includes an assessment of the equilibration process and compares the force field parameters that we developed for the azobenzene with those proposed by Böckmann et al. ${ }^{51}$

\section{Acknowledgments}

We thank the University of Nottingham and SOLEIL for a Ph.D. studentship for F.A. We are grateful for access to the University of Nottingham High Performance Computer. 


\section{References}

(1) Brown, J. E.; Klee, W. A. Helix-coil transition of the isolated amino terminus of ribonuclease. Biochemistry 1971, 10, 470-476.

(2) Scholtz, J. M.; Baldwin, R. L. The mechanism of alpha-helix formation by peptides. Ann. Rev. Biophys. Biomol. Struct. 1992, 21, 95-118.

(3) Andrew, C. D.; Bhattacharjee, S.; Kokkoni, N.; Hirst, J. D.; Jones, G. R.; Doig, A. J. Stabilizing interactions between aromatic and basic side chains in alpha-helical peptides and proteins. Tyrosine effects on helix circular dichroism. J. Am. Chem. Soc. 2002, 124, $12706-12714$.

(4) Williams, S.; Causgrove, T. P.; Gilmanshin, R.; Fang, K. S.; Callender, R. H.; Woodruff, W. H.; Dyer, R. B. Fast events in protein folding: Helix melting and formation in a small peptide. Biochemistry 1996, 35, 691-697.

(5) Lednev, I. K.; Karnoup, A. S.; Sparrow, M. C.; Asher, S. A. Alpha-helix peptide folding and unfolding activation barriers: A nanosecond UV resonance Raman study. J. Am. Chem. Soc. 1999, 121, 8074-8086.

(6) Khuc, M. T.; Mendonca, L.; Sharma, S.; Solinas, X.; Volk, M.; Hache, F. Measurement of circular dichroism dynamics in a nanosecond temperature-jump experiment. Rev. Sci. Instrum. 2011, 82, 054302.

(7) Chen, Y. H.; Yang, J. T.; Martinez, H. M. Determination of the secondary structures of proteins by circular dichroism and optical rotatory dispersion. Biochemistry 1972, 11, 4120-4131.

(8) Woody, R. W. Circular dichroism. Meth Enzymol. 1995, 246, 34-71.

(9) Christov, C.; Tielens, F.; Mirazchiiski, M. Modeling study of the influences of the 
aromatic transitions and the local environment on the far-UV rotational strengths in TEM-1 beta-lactamase. J. Mol. Model. 2006, 12, 411-416.

(10) Dang, Z.; Hirst, J. D. Short hydrogen bonds, circular dichroism and over-estimates of peptide helicity. Angew. Chemie Intl. Ed. 2001, 113, 3731-3733.

(11) Besley, N. A.; Hirst, J. D. Theoretical studies toward quantitative protein circular dichroism calculations. J. Am. Chem. Soc. 1999, 121, 9636-9644.

(12) Shea, J. E.; Brooks III, C. L. From folding theories to folding proteins: A review and assessment of simulation studies of protein folding and unfolding. Annu. Rev. Phys. Chem. 2001, 52, 499-535.

(13) Georgoulia, P. S.; Glykos, N. M. Molecular simulation of peptides coming of age: Accurate prediction of folding, dynamics and structures. Arch. Biochem. Biophys. 2019, $664,76-88$.

(14) Rogers, D. M.; Jasim, S. B.; Dyer, N. T.; Auvray, F.; Réfrègiers, M.; Hirst, J. D. Electronic circular dichroism of proteins. Chem 2019, 5, 2751-2774.

(15) Micsonai, A.; Wien, F.; Kernya, L.; Lee, Y. H.; Goto, Y.; Réfrégiers, M.; Kardos, J. Accurate secondary structure prediction and fold recognition for circular dichroism spectroscopy. Proc. Natl. Acad. Sci. U.S.A. 2015, 112, E3095-E3103.

(16) Mavridis, L.; Janes, R. W. PDB2CD: A web-based application for the generation of circular dichroism spectra from protein atomic coordinates. Bioinformatics 2017, 33, $56-63$.

(17) Nagy, G.; Igaev, M.; Jones, N. C.; Hoffmann, S. V.; Grubmuller, H. SESCA: Predicting circular dichroism spectra from protein molecular structures. J. Chem. Theor. Comput. 2019, 15, 5087-5102. 
(18) Kaminský, J.; Kubelka, J.; Bour, P. Theoretical modeling of peptide alpha-helical circular dichroism in aqueous solution. J. Phys. Chem. A 2011, 115, 1734-1742.

(19) Seibert, J.; Bannwarth, C.; Grimme, S. Biomolecular structure information from highspeed quantum mechanical electronic spectra calculation. J. Am. Chem. Soc. 2017, $139,11682-11685$.

(20) Woody, R. W. Improved Calculation of the $n \pi^{*}$ Rotational Strength in Polypeptides. J. Chem. Phys. 1968, 49, 4797-4806.

(21) Bayley, P. M.; Nielsen, E. B.; Schellman, J. A. Rotatory properties of molecules containing two peptide groups: theory. J. Phys. Chem. 1969, 73, 228-243.

(22) Ianeselli, A.; Orioli, S.; Spagnolli, G.; Faccioli, P.; Cupellini, L.; Jurinovich, S.; Mennucci, B. Atomic detail of protein folding revealed by an ab initio reappraisal of circular dichroism. J. Am. Chem. Soc. 2018, 140, 3674-3682.

(23) Bulheller, B. M.; Hirst, J. D. DichroCalc - circular and linear dichroism online. Bioinformatics 2009, 25, 539-540.

(24) Hildebrand, N.; Michaelis, M.; Wurzler, N.; Li, Z.; Hirst, J. D.; Micsonai, A.; Kardos, J.; Gil-Ley, A.; Bussi, G.; Koppen, S., et al. Atomistic details of chymotrypsin conformational changes upon adsorption on silica. ACS Biomater. Sci. Eng. 2018, 4, 4036-4050.

(25) Michaelis, M.; Hildebrand, N.; Meißner, R. H.; Wurzler, N.; Li, Z.; Hirst, J. D.; Micsonai, A.; Kardos, J.; Delle Piane, M.; Colombi Ciacchi, L. Impact of the conformational variability of oligopeptides on the computational prediction of their CD spectra. J. Phys. Chem. B 2019, 123, 6694-6704.

(26) First, J. T.; Webb, L. J. Agreement between experimental and simulated circular 
dichroic spectra of a positively charged peptide in aqueous solution and on selfassembled monolayers. J. Phys. Chem. B 2019, 123, 4512-4526.

(27) Gattuso, H.; Garcia-Iriepa, C.; Sampedro, D.; Monari, A.; Marazzi, M. Simulating the electronic circular dichroism spectra of photoreversible peptide conformations. $J$. Chem. Theor. Comput. 2017, 13, 3290-3296.

(28) Szymański, W.; Beierle, J. M.; Kistemaker, H. A. V.; Velema, W. A.; Feringa, B. L. Reversible photocontrol of biological systems by the incorporation of molecular photoswitches. Chem. Rev. 2013, 113, 6114-6178.

(29) Woolley, G. A. Photocontrolling peptide $\alpha$ helices. Acc. Chem. Res. 2005, 38, 486-493.

(30) Beharry, A. A.; Woolley, G. A. Azobenzene photoswitches for biomolecules. Chem. Soc. Rev. 2011, 40, 4422-4437.

(31) Zhao, Y.; Ikeda, T. Smart light-responsive materials: azobenzene-containing polymers and liquid crystals.; John Wiley \& Sons, 2009.

(32) Nenov, A.; Borrego-Varillas, R.; Oriana, A.; Ganzer, L.; Segatta, F.; Conti, I.; SegarraMarti, J.; Omachi, J.; Dapor, M.; Taioli, S., et al. UV-light-induced vibrational coherences: the key to understand Kasha rule violation in trans-azobenzene. J. Phys. Chem. Lett. 2018, 9, 1534-1541.

(33) Nägele, T.; Hoche, R.; Zinth, W.; Wachtveitl, J. Femtosecond photoisomerization of cis-azobenzene. Chem. Phys. Lett. 1997, 272, 489-495.

(34) Fujino, T.; Tahara, T. Picosecond time-resolved Raman study of trans-azobenzene. J. Phys. Chem. A 2000, 104, 4203-4210.

(35) Fujino, T.; Arzhantsev, S. Y.; Tahara, T. Femtosecond time-resolved fluorescence study of photoisomerization of trans-azobenzene. J. Phys. Chem. A 2001, 105, 8123-8129. 
(36) Renner, C.; Moroder, L. Azobenzene as conformational switch in model peptides. ChemBioChem 2006, 7, 868-678.

(37) Spörlein, S.; Carstens, H.; Satzger, H.; Renner, C.; Behrendt, R.; Moroder, L.; Tavan, P.; Zinth, W.; Wachtveit, J. Ultrafast spectroscopy reveals subnanosecond peptide conformational dynamics and validates molecular dynamics simulation. Proc. Natl. Acad. Sci. U.S.A. 2002, 99, 7998-8002.

(38) Buchli, B.; A., W. S.; Walser, R.; Donten, M. L.; Pfister, R.; Blöchliger, N.; Steiner, S.; Caflisch, A.; Zerbe, O.; Hamm, P. Kinetic response of a photoperturbed allosteric protein. Proc. Natl. Acad. Sci. U.S.A. 2013, 110, 11725-11730.

(39) Flint, D. G.; Kumita, J. R.; Smart, O. S.; Woolley, G. A. Using an azobenzene crosslinker to either increase or decrease peptide helix content upon trans-to-cis photoisomerization. Chem. Biol. 2002, 9, 391-397.

(40) Chen, E.; Kumita, J. R.; Woolley, G. A.; Kliger, D. S. The kinetics of helix unfolding of an azobenzene cross-linked peptide probed by nanosecond time-resolved optical rotatory dispersion. J. Am. Chem. Soc. 2003, 125, 12443-12449.

(41) Bredenbeck, J.; Helbing, J.; Kumita, J. R.; Woolley, G. A.; Hamm, P. $\alpha$-Helix formation in a photoswitchable peptide tracked from picoseconds to microseconds by time-resolved IR spectroscopy. Proc. Natl. Acad. Sci. U.S.A. 2005, 102, 2379-2384.

(42) Xia, S. H.; Cui, G.; Fang, W. H.; Thiel, W. How photoisomerization drives peptide folding and unfolding: Insights from QM/MM and MM dynamics simulations. Angew. Chem. Int. Ed. 2016, 128, 2107-2112.

(43) Hanwell, M. D.; Curtis, D. E.; Lonie, D. C.; Vandermeersch, T.; Zurek, E.; Hutchison, G. R. Avogadro: an advanced semantic chemical editor, visualization, and analysis platform. J. Cheminformatics 2012, 4, 17. 
(44) Humphrey, W.; Dalke, A.; Schulten, K. VMD: visual molecular dynamics. J. Mol. Graph. 1996, 14, 33-38.

(45) Jorgensen, W. L.; Chandrasekhar, J.; Madura, J. D.; Impey, R. W.; Klein, M. L. Comparison of simple potential functions for simulating liquid water. J. Chem. Phys. 1983, 79, 926-935.

(46) Phillips, J. C.; Braun, R.; Wang, W.; Gumbart, J.; Tajkhorshid, E.; Villa, E.; Chipot, C.; Skeel, R. D.; Kale, L.; Schulten, K. Scalable molecular dynamics with NAMD. J. Comput. Chem. 2005, 26, 1781-1802.

(47) MacKerell Jr, A. D.; Bashford, D.; Bellott, M.; Dunbrack Jr, R. L.; Evanseck, J. D.; Field, M. J.; Fischer, S.; Gao, J.; Guo, H.; Ha, S., et al. All-atom empirical potential for molecular modeling and dynamics studies of proteins. J. Phys. Chem. 1998, 102, $3586-3616$.

(48) MacKerell Jr, A. D.; Feig, M.; Brooks, C. L. Improved treatment of the protein backbone in empirical force fields. J. Am. Chem. Soc. 2003, 126, 698-699.

(49) Best, R. B.; Zhu, X.; Shim, J.; Lopes, P. E.; Mittal, J.; Feig, M.; MacKerell Jr, A. D. Optimization of the additive CHARMM all-atom protein force field targeting improved sampling of the backbone $\phi, \psi$ and side-chain $\chi^{1}$ and $\chi^{2}$ dihedral angles. J. Chem. Theory Comput. 2012, 8, 3257-3273.

(50) Carstens, H. Konformationsdynamik lichtschaltbarer peptide: Molekulardynamiksimulationen und datengetriebene modellbildung [Conformational dynamics of photoswitchable peptides: Molecular dynamics and data-driven model building]. PhD thesis (Ludwig Maximilians Universität München, Munich). Available at: http://edoc.ub.unimuenchen.de/2268. 2004,

(51) Böckmann, M.; Peter, C.; Site, L. D.; Doltsinis, N. L.; Kremer, K.; Marx, D. Atomistic 
force field for azobenzene compounds adapted for QM/MM simulations with applications to liquids and liquid crystals. J. Chem. Theory Comput. 2007, 3, 1789-1802.

(52) Vanommeslaeghe, K.; MacKerell Jr, A. D. Automation of the CHARMM General Force Field (CGenFF) I: bond perception and atom typing. J. Chem. Inf. Model. 2012, 52, $3144-3154$.

(53) Vanommeslaeghe, K.; Hatcher, E.; Acharya, C.; Kundu, S.; Zhong, S.; Shim, J.; Darian, E.; Guvench, O.; Lopes, P.; Vorobyov, I., et al. CHARMM general force field: A force field for drug-like molecules compatible with the CHARMM all-atom additive biological force fields. J. Comput. Chem. 2010, 31, 671-690.

(54) Ihalainen, J. A.; Bredenbeck, J.; Pfister, R.; Helbing, J.; Chi, L.; van Stokkum, I. H.; Woolley, G. A.; Hamm, P. Folding and unfolding of a photoswitchable peptide from picoseconds to microseconds. Proc. Natl. Acad. Sci. U.S.A. 2007, 104, 5383-5388.

(55) Shao, Y.; Gan, Z.; Epifanovsky, E.; Gilbert, A. T.; Wormit, M.; Kussmann, J.; Lange, A. W.; Behn, A.; Deng, J.; Feng, X., et al. Advances in molecular quantum chemistry contained in the Q-Chem 4 program package. Mol. Phys. 2015, 113, 184215.

(56) Feller, S. E.; Pastor, R. W.; Rojnuckarin, A.; Bogusz, S.; Brooks, B. R. Effect of electrostatic force truncation on interfacial and transport properties of water. J. Phys. Chem. 1996, 100, 17011-17020.

(57) Miyamoto, S.; Kollman, P. A. Settle: An analytical version of the SHAKE and RATTLE algorithm for rigid water models. J. Comput. Chem. 1992, 13, 952-962.

(58) Ryckaert, J. P.; Ciccotti, G.; Berendsen, H. J. Numerical integration of the cartesian equations of motion of a system with constraints: molecular dynamics of n-alkanes. $J$. Comput. Phys. 1977, 23, 327-341. 
(59) Paoli, B.; Seeber, M.; Backus, E. H.; Ihalainen, J. A.; Hamm, P.; Caflisch, A. Bulky side chains and non-native salt bridges slow down the folding of a cross-linked helical peptide: a combined molecular dynamics and time-resolved infrared spectroscopy study. J. Phys. Chem. B 2009, 113, 4435-4442.

(60) Amadei, A.; Ceruso, M. A.; Di Nola, A. On the convergence of the conformational coordinates basis set obtained by the essential dynamics analysis of proteins' molecular dynamics simulations. Proteins Str. Func. Bioinf. 1999, 36, 419-424.

(61) Bakan, A.; Meireles, L. M.; Bahar, I. ProDy: protein dynamics inferred from theory and experiments. Bioinformatics 2011, 27, 1575-1577.

(62) Spek, E. J.; Olson, C. A.; Shi, Z.; Kallenbach, N. R. Alanine is an intrinsic $\alpha$-helix stabilizing amino acid. J. Am. Chem. Soc. 1999, 121, 5571-5572.

(63) Hollingsworth, S. A.; Karplus, P. A. A fresh look at the Ramachandran plot and the occurrence of standard structures in proteins. Biomol. Concepts 2010, 1, 271-283.

(64) Hirst, J. D.; Brooks III, C. L. Molecular dynamics simulations of isolated helices of myoglobin. Biochemistry 1995, 34, 7614-7621.

(65) Auvray, F.; Dennetiere, D.; Giuliani, A.; Jamme, F.; Wien, F.; Nay, B.; Zirah, S.; Polack, F.; Menneglier, C.; Lagarde, B.; Hirst, J. D.; Réfrégiers, M. Time resolved transient circular dichroism spectroscopy using synchrotron natural polarization. Struct. Dynam. 2019, 6, 054307.

(66) Dartigalongue, T.; Niezborala, C.; Hache, F. Subpicosecond UV spectroscopy of carbonmonoxy-myoglobin: absorption and circular dichroism studies. Phys. Chem. Chem. Phys. 2007, 9, 1611-1615. 


\section{Supporting Information}

\section{Unfolding Dynamics of a Photo-switchable Helical Peptide}

Francois Auvray and Jonathan D. Hirst

\section{Equilibration RMSD}

The averaged root-mean-squared deviation (RMSD) of the peptide backbone is plotted as a function of time in Figure S1.

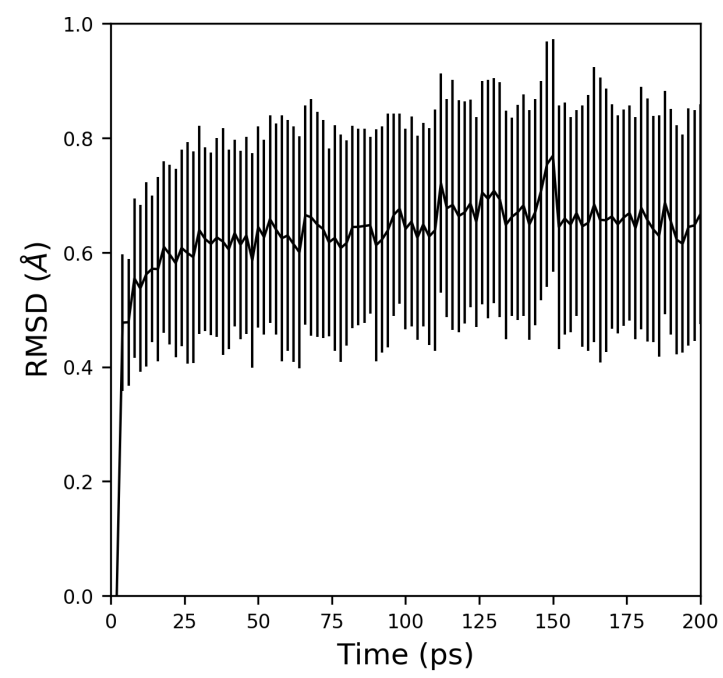

Figure S1: RMSD variation over time. The error bars width corresponds to one standard deviation. The RMSD calculation is only base on the atoms of the peptide backbone.

The reference of our RMSD calculation was the initial conformation of the equilibration simulation. The RMSD increases over the first 25 ps of the simulation and then stabilises around $0.65 \AA$, showing that the backbone structures reach stable conformations and that the systems are equilibrated. 


\section{Force field parameters}

The labeled structures of the parameterised molecules are shown in Figure S2. The force field parameters proposed by Böckmann et al. ${ }^{51}$ for the azobenzene (Figure S2a) are shown in Table S1. The optimised parameters presented in this paper for the structure shown in Figure S2a and S2b are in Table S2 and S3, respectively.

a

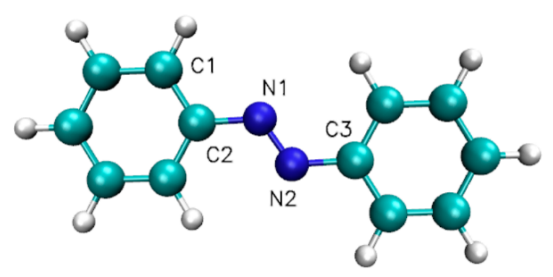

b

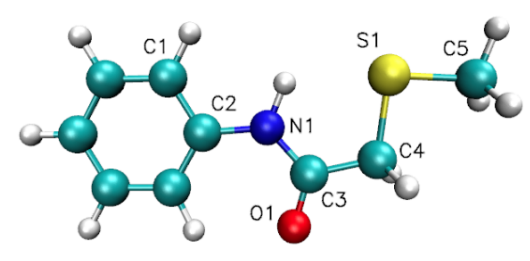

Figure S2: a. Azobenzene b. Structures used for the force field parameter optimisation of the linker between the azobenzene and the peptide.

Table S1: Optimised force field parameters of the azobenzene (Figure S2a) proposed by Böckmann et al. ${ }^{51}$

\begin{tabular}{|c|c|c|c|c|c|c|c|c|c|}
\hline Dihedral angle & $k(\mathrm{kcal} / \mathrm{mol})$ & $n$ & $\delta\left(^{\circ}\right)$ & Angle & $k(\mathrm{kcal} / \mathrm{mol})$ & $\theta_{0}\left(^{\circ}\right)$ & Bond & $k(\mathrm{kcal} / \mathrm{mol})$ & $b_{0}(\AA)$ \\
\hline $\mathrm{C}_{1} \mathrm{C}_{2} \mathrm{~N}_{1} \mathrm{~N}_{2}$ & 1.43 & 2 & 180 & $\mathrm{C}_{1} \mathrm{C}_{2} \mathrm{~N}_{1}$ & 134 & 120.0 & $\mathrm{C}_{2} \mathrm{~N}_{1}$ & 172 & 1.432 \\
\hline $\mathrm{C}_{2} \mathrm{~N}_{1} \mathrm{~N}_{2} \mathrm{C}_{3}$ & 16.70 & 2 & 180 & $\mathrm{C}_{2} \mathrm{~N}_{1} \mathrm{~N}_{2}$ & 155 & 116.5 & $\mathrm{~N}_{1} \mathrm{~N}_{2}$ & 335 & 1.262 \\
\hline
\end{tabular}

Table S2: Optimised force field parameters of the azobenzene (Figure S2a).

\begin{tabular}{lccc}
\hline Dihedral angle & $k(\mathrm{kcal} / \mathrm{mol})$ & $n$ & $\delta\left(^{\circ}\right)$ \\
\hline $\mathrm{C}_{1} \mathrm{C}_{2} \mathrm{~N}_{1} \mathrm{~N}_{2}$ & 2.5 & 2 & 180 \\
$\mathrm{C}_{2} \mathrm{~N}_{1} \mathrm{~N}_{2} \mathrm{C}_{3}$ & 23.0 & 2 & 180 \\
\hline
\end{tabular}

\begin{tabular}{lcc}
\hline Angle & $k(\mathrm{kcal} / \mathrm{mol})$ & $\theta_{0}\left(^{\circ}\right)$ \\
\hline $\mathrm{C}_{1} \mathrm{C}_{2} \mathrm{~N}_{1}$ & 50 & 116.5 \\
$\mathrm{C}_{2} \mathrm{~N}_{1} \mathrm{~N}_{2}$ & 75 & 106.0
\end{tabular}

\begin{tabular}{lcc}
\hline Bond & $k(\mathrm{kcal} / \mathrm{mol})$ & $b_{0}(\AA)$ \\
\hline $\mathrm{C}_{2} \mathrm{~N}_{1}$ & 300 & 1.415 \\
$\mathrm{~N}_{1} \mathrm{~N}_{2}$ & 500 & 1.295 \\
\hline
\end{tabular}



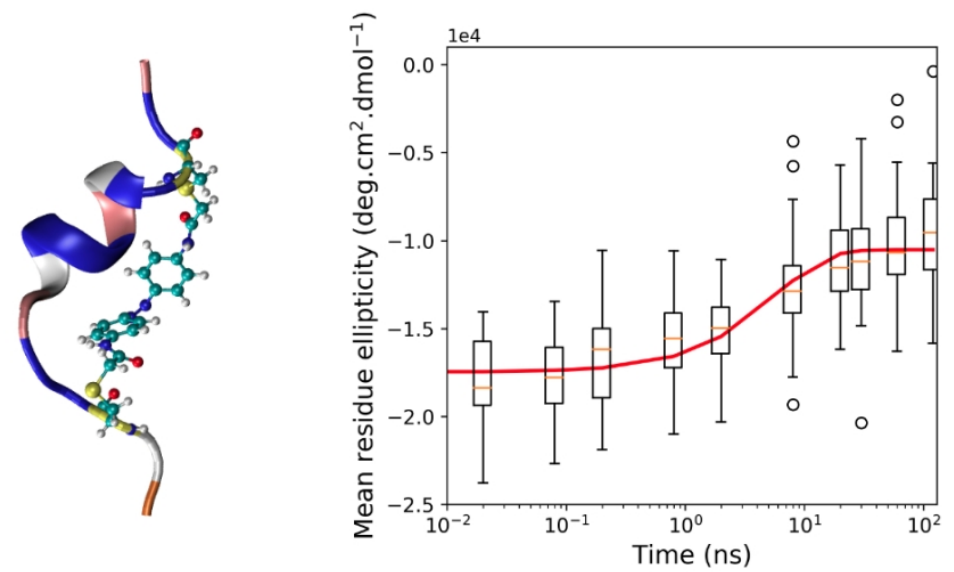

TOC graphic $338 \times 190 \mathrm{~mm}(96 \times 96$ DPI) 